\title{
AN ULTRASONICALLY POWERED IMPLANTABLE MICRO ELECTROLYTIC ABLATION (IMEA) FOR TUMOR NECROSIS
}

\author{
Anirvan K. Majumdar ${ }^{1}$, Sayemul Islam ${ }^{l}$, and Albert Kim ${ }^{1 *}$
}

${ }^{1}$ Department of Electrical Engineering, Temple University, Philadelphia, Pennsylvania, USA

\begin{abstract}
Electrolytic ablation is a technique that can remove nonresectable tumors from internal organs (such as liver, kidney, pancreas, etc.) with highly localized control to minimize harm to adjoining healthy tissue. Here, we aim to utilize the principle of electrolytic ablation in an implantable platform and power it by an external ultrasonic wave. The implantable micro electrolytic ablation (IMEA) will address challenges of the current existing tethered method such as constraints in electrode size, multiple targets and repeated treatments in case of cancer recurrence. We characterized the prototype of IMEA in an agarose gel containing phenolphthalein to simulate internal body tissue. Color change in phenolphthalein shows that the device responds to external ultrasonic stimulation and shows electrolytic behavior in an area around the electrodes that spreads outward with time. Overall, the IMEA could achieve $0.614 \pm 0.01 \mathrm{~cm}^{2}$ in ablation area (cathode) when $\sim 190 \mathrm{~mW} / \mathrm{cm}^{2}$ ultrasonic intensity was applied for $60 \mathrm{~min}$.
\end{abstract}

\section{INTRODUCTION}

Pancreatic ductal adenocarcinoma accounts for about $80 \%$ of all malignant pancreatic tumors [1] with an unusually low 5-year survival rate. This is attributed to its rapid growth, aggressive malignancy, and insidious course [2]. These factors are also responsible for the low rates of early detection, which in turn leads to low rates of survival since most of the cases that are finally diagnosed are only done so at late stages [2]. Surgically resectable tumors are observed in only $20 \%$ of the diagnosed patients [1]. Chemotherapy and radiotherapy are applied to the remaining nonresectable cases [1]. More recently, ablation technology has become available for locally advanced unresectable non-metastatic tumors $[1,2]$.

Ablation is a minimally invasive technique that uses either thermal or electric energy to cause localized necrosis of tissue. Unfortunately, current ablation technology uses externally inserted electrodes to cause localized cell death within the body. The electrode size becomes a constraint in trying to maximize stiffness (so that it doesn't snap off within the body) and simultaneously minimizing ablation area (to avoid death of neighboring healthy cells). Multiple tumors may require multiple incisions. Moreover, in cases of cancer recurrence, the entire procedure will have to be repeated.

In this paper, we report a novel implantable micro electrolytic ablation device (IMEA) that can be used to perform electrolytic ablation while being powered by an external ultrasound source. It is expected that the IMEA will alleviate some of the issues faced by conventional ablation techniques.

\section{MATERIALS AND METHODS}

Figure 1.a shows a schematic view of Implantable Micro Electrolytic Ablation (IMEA). The ultrasonic wave can reach a deeply implanted IMEA device (such as, near the pancreas). The ultrasonic wave is generated from an ultrasonic transducer that is excited by a signal generator via a power amplifier. The ultrasonic wave travels through tissue and reach an implanted IMEA that consists of an ultrasonic receiver, rectifier, and platinum wires (Figure 1.b). The ultrasonic wave is being transformed to electrical power through piezoelectricity, providing sufficient potential for electrolysis. In physiological medium, electrochemical reactions by electrolysis yield changes in $\mathrm{pH}$ due to newly generated chemical species (anode: $2 \mathrm{H}_{2} \mathrm{O} \rightarrow \mathrm{O}_{2}+4 \mathrm{H}^{+}+4 e^{-}$, cathode: $2 \mathrm{H}_{2} \mathrm{O}+2 e^{-} \rightarrow$ $\mathrm{H}_{2}+2 \mathrm{OH}^{-}$). Therefore, local cytotoxic regions are formed that diffuse around the electrodes [3-5].

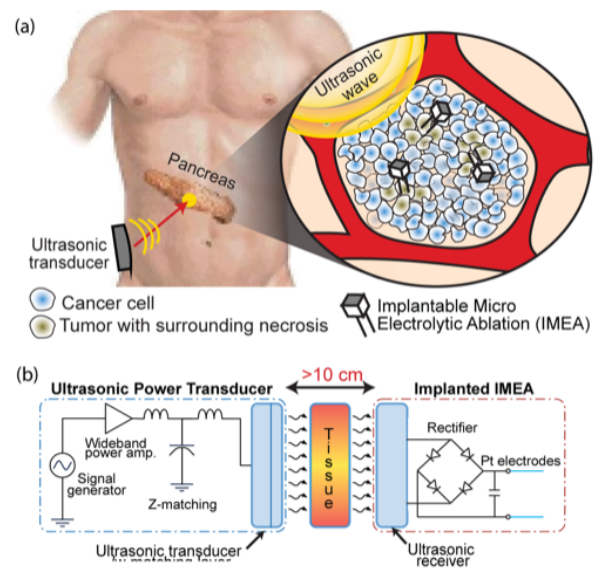

Figure 1: (a) IMEA implanted in a pancreatic tumor for in situ ablation, (b) schematic of ultrasonic system used to power the IMEA

Figure 2 shows the fabrication process. The entire electronics is housed on a flexible PCB made by chemical etching of a copperclad laminated sheet (Pyralux ${ }^{\circledR}$, DuPont) on which the routing is drawn (Figure 2.a-b). After the circuit components are soldered (Figure 2.c-d), passivation is done by epoxy, and a custom capsule is $3 \mathrm{D}$ printed to fit the device (Figure 2.e). Figure 3 shows a picture of the final prototype of IMEA. Platinum electrodes are approximately $9 \mathrm{~mm}$ in length for both anode and cathode.

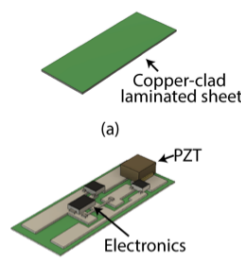

(c)

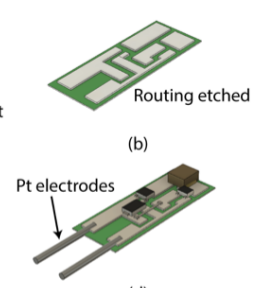

(d)

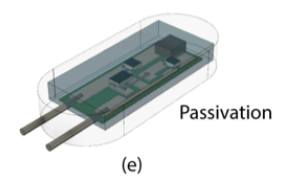

(e)
Figure 2: IMEA fabrication process: (a) circuit is drawn on Pyralux, (b) copper etching, (c) electronics integration, (d) platinum electrodes integration, and (e) passivation and packaging

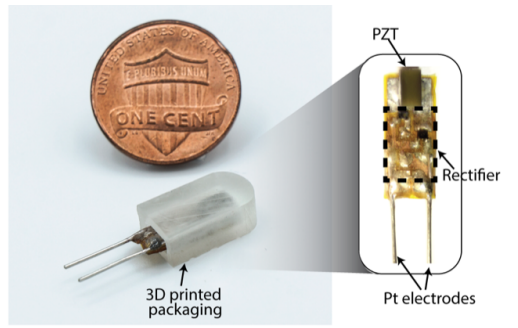

Figure 3: Final prototype of the IMEA showing the device within the packaging as well as without. Electrodes length is approximately 9mm Hilton Head Island, South Carolina, June 3-7, 2018 


\section{RESULTS AND DISCUSSION}

Figure 4 shows the experimental setup. A function generator is used to generate a signal that is amplified by an RF amplifier and converted to ultrasound by a piezoelectric slab (Lead Zirconate Titanate, PZT5H, Piezo Inc.). The ultrasound is transmitted to the IMEA through the agarose. The piezoelectric receiver on the device receives the ultrasonic wave and produces $\mathrm{AC}$ power. This is passed through a full-wave rectifier to be converted to DC power. The DC power is finally fed to attached platinum electrodes (cathode and anode) which cause localized $\mathrm{pH}$ change by electrolysis.

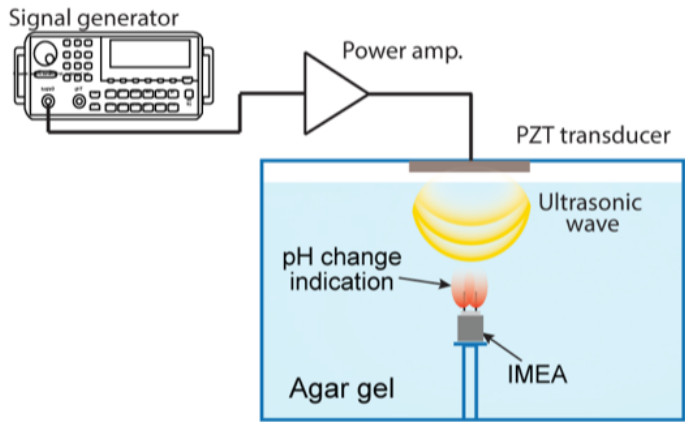

Figure 4: Experimental setup for in vitro characterization of the IMEA operation. The IMEA was placed in agarose gel with $\mathrm{pH}$ sensitive dye while it is powered by an ultrasonic transducer

Phenolphthalein dye-containing agarose gels are prepared to simulate tissue environment. The agarose gel is formed using $0.7 \mathrm{wt} . \%$ agarose and $0.9 \mathrm{wt} . \%$ sodium chloride. $1 \mathrm{ml}$ of phenolphthalein is added per $100 \mathrm{ml}$ of agarose solution. A central crater is chiseled out in the gel to place the device and the electrodes are inserted into the gel leaving the PZT receiver and circuit body in the crater. The crater is filled with deionized water for ultrasonic wave propagation. The ultrasonic transducer (i.e. the PZT slab used to generate the ultrasound) is placed on top of the crater. The signal generator is adjusted to a frequency of $1.15 \mathrm{MHz}$ (the resonant frequency of the $2 \mathrm{~mm}$-thick PZT). While various input voltage amplitude $(50,75$, and $100 \mathrm{mVp}-\mathrm{p})$ were applied, corresponding $\mathrm{pH}$ changes were analyzed by characterizing the color change of the gel. Note that the ultrasound intensity was calibrated by a fiber optics hydrophone (Precision Acoustic Ltd.). Input voltage of 50, 75 and $100 \mathrm{mVp}$-p input corresponded to maximum acoustic intensity of approximately 46,119 , and $190 \mathrm{~mW} / \mathrm{cm}^{2}$, respectively.

As ultrasound is applied to the device, a colored region (theoretical ablation region) is observed around the electrode in response to changing $\mathrm{pH}$. Images are captured at an interval of 5 min over lhour. The ablation region was measured by image processing using ImageJ. The ablation region around the cathode increases with time until it shows a plateauing (Figure 5). The ablation area around the anode was observed to be smaller than that around the cathode (Figure 6). Ideally, the anode should be inactive, but since the circuit does not have an earth ground, it remains active. Increasing the ultrasonic power decreases the time taken to reach maximum ablation area, though the relationship is not linear.

\section{CONCLUSIONS}

We present here an ultrasonically powered implantable micro electrolytic ablation device intended for tumor ablation. In vitro results show effective $\mathrm{pH}$ change in agar gel that could possibly translate into appropriate in vivo tissue necrosis. It is expected that this device alleviates some of the drawbacks of conventional electrolytic ablation by decreasing the number of procedures required in case of cancer recurrences.

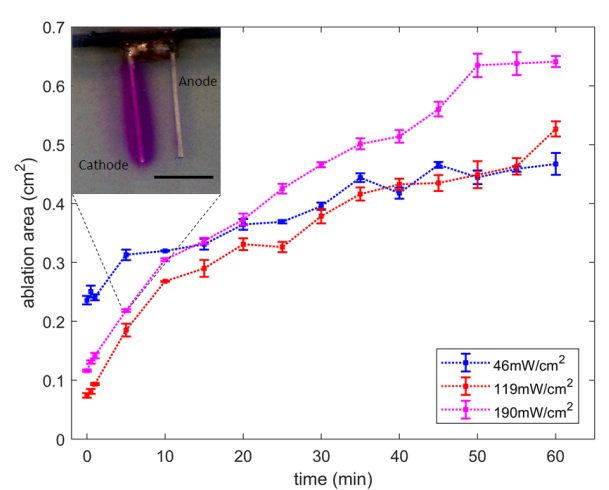

Figure 5: The time-dependent increase in the ablation area of the cathode when supplied with $46 \mathrm{~mW} / \mathrm{cm}^{2}$ (blue), $119 \mathrm{~mW} / \mathrm{cm}^{2}$ (red) and $190 \mathrm{~mW} / \mathrm{cm}^{2}$ (magenta). The insert shows a sample image highlighting the cathode at 5 min time point when the IMEA is supplied with $190 \mathrm{~mW} / \mathrm{cm}^{2}$. Insert scale bar represents $5 \mathrm{~mm}$.

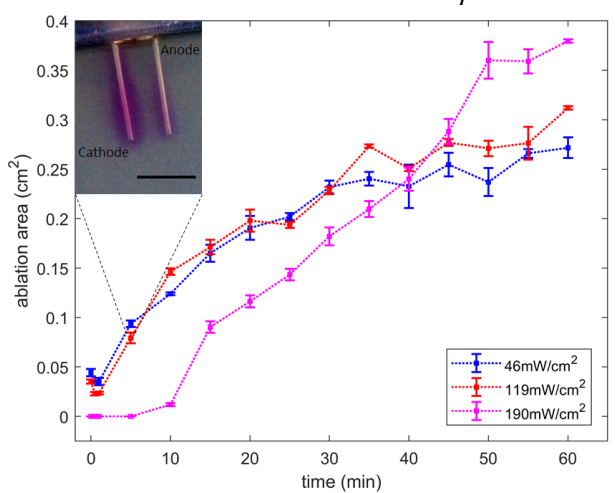

Figure 6: The time-dependent increase in the ablation area of the anode when supplied with $46 \mathrm{~mW} / \mathrm{cm}^{2}$ (blue), $119 \mathrm{~mW} / \mathrm{cm}^{2}$ (red) and $190 \mathrm{~mW} / \mathrm{cm}^{2}$ (magenta). The insert shows a sample image highlighting the anode at $5 \mathrm{~min}$ time point when the IMEA is supplied with $119 \mathrm{~mW} / \mathrm{cm}^{2}$. Insert scale bar represents $5 \mathrm{~mm}$.

\section{REFERENCES}

[1] D’Onofrio, M., Ciaravino, V., De Robertis, R., Barbi, E., Salvia, R., Girelli, R., Paiella, S., Gasparini, C., Cardobi, N. and Bassi, C., "Percutaneous ablation of pancreatic cancer", World journal of gastroenterology, 22, 44 (1992), pp. 9661.

[2] Hadjicostas, P., Malakounides, N., Varianos, C., Kitiris, E., Lerni, F. and Symeonides, P., "Radiofrequency ablation in pancreatic cancer", HPB, 8, 1 (2006), pp. 61-64.

[3] Stehling, M.K., Guenther, E., Mikus, P., Klein, N., Rubinsky, L. and Rubinsky, B., "Synergistic combination of electrolysis and electroporation for tissue ablation", PLOS One, 11, 2 (2016), pp. e0148317.

[4] Phillips, M., Krishnan, H., Raju, N. and Rubinsky, B., "Tissue ablation by a synergistic combination of electroporation and electrolysis delivered by a single pulse", Annals of biomedical engineering, 44, 10 (2016), pp. 3144-3154.

[5] Lugnani, F., Macchioro, M. and Rubinsky, B., "Cryoelectrolysis-electrolytic processes in a frozen physiological saline medium”, PeerJ, 5 (2017), p. e2810.

\section{ACKNOWLEDGEMENTS}

We thank Dr. Iyad Obeid for his support and valuable insights.

\section{CONTACT}

*Prof. Albert Kim, albertkim@temple.edu 